BMJ

Open

Gastroenterology

\title{
Non-alcoholic fatty liver disease (NAFLD): a significant predictor of gestational diabetes mellitus (GDM) and early pregnancy miscarriages - prospective study in Rajarata Pregnancy Cohort (RaPCo)
}

Iresha Sandamali Koralegedara (i) , 1,2,3 Janith Niwanthaka Warnasekara, ${ }^{2,3}$ Korale Gedara Dayaratne, ${ }^{4}$ Farika Nirmani De Silva, ${ }^{2,3}$ Jagath Keerthi Premadasa, ${ }^{5}$ Suneth Buddhika Agampodi ${ }^{2,3}$

\section{ABSTRACT}

Background and aims Non-alcoholic fatty liver disease (NAFLD) is increasing globally with a mounting body of evidence on various adverse effects on pregnancy. Yet, prospective studies, especially from low-income and middle-income countries, are lacking in examining the impact of NAFLD in pregnancy. In this study, we explored the effect of NAFLD on the development of gestational diabetes mellitus (GDM) and early pregnancy miscarriages. Methods A population-based prospective cohort study was conducted among first-trimester pregnant women who registered in the national pregnancy care programme during July-September 2019 in Anuradhapura district, Sri Lanka. Baseline clinical-biochemical parameters and ultrasound scan (USS) of the liver were done to assess fatty liver. Those who were normoglycaemic based on WHO criteria were followed up, and a repeat oral glucose tolerance test was performed between 24 and 28 weeks of gestation.

Results Of the 632 pregnant women studied, 90 (14\%) and $234(37 \%)$ were diagnosed as having fatty liver grade (FLG) II and I, respectively. The cumulative incidence of GDM in FLG 0, I, and II were 11, 44, and 162 per 1000 pregnancies, respectively. After adjusting for age and other known risk factors, women with FLG II had a relative risk (RR) of $12.5(95 \% \mathrm{Cl} 2.2$ to 66.4$)$ for developing GDM compared with FLG 0 . In addition, women with FLG I (RR 2.1, 95\% Cl 1.01 to 4.64) and FLG II (RR 4.5, 95\% Cl 2.1 to 9.9) were significant risk factors for early pregnancy miscarriages, and FLG II remained as the only independent predictor of miscarriages after adjusting for age, parity, body mass index, blood sugar, blood pressure, and haemoglobin level (adjusted OR 4.2 (95\% $\mathrm{Cl} 1.9$ to 9.1$)$ ).

Conclusion In this rural south Asian community, NAFLD is shown to be a major risk factor for GDM and early pregnancy miscarriages. Therefore, routine identification of NAFLD through a simple USS may help in the early identification of high-risk mothers.

\section{Summary box}

What is already known about this subject?

- Although there are proven adverse pregnancy outcomes due to non-alcoholic fatty liver disease (NAFLD), it is not routinely recognised in early pregnancy.

- There are no adequate prospective evidence to support the association of NAFLD with gestational diabetes mellitus (GDM) and miscarriages in South Asian community.

What are the new findings?

- Fatty liver grade II is shown to be an independent predictor of GDM and early pregnancy miscarriages.

How might it impact on clinical practice in the foreseeable future?

- Routine identification of NAFLD through simple non-invasive ultrasound scan may help in early identification of high-risk mothers, hence early interventions to improve maternal morbidity.

\section{INTRODUCTION}

Non-alcoholic fatty liver disease (NAFLD) is an initial manifestation of various pathological conditions, such as non-alcoholic steatohepatitis, cirrhosis and liver malignancies. ${ }^{\prime}$ The most implicated mechanism for NAFLD is insulin resistance. ${ }^{2}$ In the two-hit hypothesis, supra-physiological levels of glucose, sucrose, and fructose can induce lipogenic genes through various mechanisms that lead to de novo lipogenesis and inhibit fatty acid oxidation, causing deposition of fatty acids in various organs such as the liver. ${ }^{3}$ The multiple-hit hypothesis describes several factors, 
including insulin resistance, hormones secreted by the adipocytes, nutritional factors, gut microbiota and genetic factors responsible for the pathogenesis of NAFLD.

A recent study shows that the prevalence of NAFLD is high in the Asian context due to increasing urbanisation leading to an epidemic of obesity. ${ }^{4}$ The pooled regional incidence of NAFLD in Asian countries is 52 per 1000 person-years compared with 28 per 1000 person-years in the West. ${ }^{5}$ The prevalence of lean NAFLD in Asia is $19 \%$, while it is $7 \%$ in the USA. ${ }^{6}$

Although NAFLD is described as the most common liver disease in the West, its effect on pregnancy has not been discussed widely until recently. ${ }^{7}$ Early retrospective studies have reported a low prevalence (28.9/per 100000 pregnancies) on NAFLD during pregnancy, ${ }^{8}$ yet with definitive adverse pregnancy outcomes. Recent studies show a varying degree of NAFLD among pregnant women, with $15 \%$ in Canada, ${ }^{10} 14.3 \%-16.7 \%$ in the USA, ${ }^{11}{ }^{12} 18.4 \%$ in Korea, ${ }^{13}$ and $18.2 \%$ in Sri Lanka. ${ }^{14}$ The secondary data analysis of the US inpatient sample of 18574225 pregnancies shows that the prevalence of NAFLD after 20 weeks of gestation has tripled over a period of 10 years. ${ }^{15}$ Since its first report in 2011, NAFLD has been identified as a major predictor of many fetal and maternal adverse outcomes, including miscarriages, ${ }^{16}$ gestational diabetes mellitus (GDM) ${ }^{13}{ }^{17-19}$ hypertensive complications, ${ }^{14}{ }^{20}$ higher caesarean sections, ${ }^{14}$ intrahepatic cholestasis in pregnancy ${ }^{21}$ preterm birth, ${ }^{20}$ low birth weight ${ }^{21}$ and postpartum haemorrhage. ${ }^{8}$

Being an insulin-resistant state, pregnancy itself has a higher risk for NAFLD as well as developing hyperglycaemia. Thus, one of the main adverse pregnancy outcomes associated with NAFLD is GDM. The pooled global prevalence of GDM using International Association of Diabetes and Pregnancy Study Group (IADPSG) criteria is reported as $10.6 \%$ (95\% CI $10.5 \%$ to $10.6 \%),{ }^{22}$ whereas the estimates for 2005-2015 shows a wide disparity across WHO regions ranging from $5.8 \%$ in Europe to 12.9 in the Middle East and North Africa. The incidence of GDM among pregnant women with NAFLD was shown to be more than $20 \%,{ }^{13}$ and the severity of NAFLD is proportional to the risk of GDM and large for gestational age babies. ${ }^{23}$ The unconfounded effect of NAFLD on GDM was estimated with an OR around 2 in two prospective cohort studies; one with OR 2.50 (95\% CI 1.07 to 5.77$)^{13}$ and another with an OR $2.2(95 \% \text { CI } 1.1 \text { to } 4.3)^{10}$ and 6.5 (95\% CI 2.3 to 18.5$)$ in another study. While early pregnancy NAFLD is almost established as a major predictor of GDM, only a limited number of prospective studies are available in global literature and none from the South Asian region, a region having a high incidence of both NAFLD and GDM. According to our knowledge, none of the prospective studies are available globally about the association between NAFLD and miscarriages.

\section{METHODOLOGY}

Aim

The purpose of the present study was to determine the role of NAFLD as a risk factor for GDM and early pregnancy miscarriages among Sri Lankan pregnant women.

\section{Study design and setting}

This study was carried out as a part of a large populationbased prospective cohort study: the Rajarata Pregnancy Cohort (RaPCo). The detailed study design of RaPCo is published elsewhere. ${ }^{24}$ The study was carried out in the Anuradhapura district, the geographically largest district in Sri Lanka. The resident population in the district is 929539, and in 2019, 15811 pregnant women registered with the national maternal care programme. Of them, 12984 were registered in field clinics before eight (8) weeks of gestation, 2063 were registered in field clinic visits at 8-12 weeks of gestation, and $98.6 \%$ had at least one clinic visit before delivery. ${ }^{25}$

The minimal sample size to estimate the prevalence of fatty liver was 405 . This was based on $95 \%$ CI, 0.03 of absolute precision, and a previously reported minimum prevalence of $10 \%$. Data published by Lee $e t a l^{23}$ were used to sample size calculation of the cohort study according to the formula published by Kelsey et al. ${ }^{26}$ The minimum sample size for cohort study with $95 \%$ confidence and $80 \%$ power was 543 .

\section{Baseline assessment}

Figure 1 is an overview of the study methodology. Pregnant women with a period of gestation of less than 12 weeks who registered with public health midwives from the end of July to September 2019 were recruited from all medical officers of health areas in Anuradhapura district.

A detailed clinical review, anthropometric measurements, and biochemical tests were performed for baseline assessment. The sociodemographic data collection was done by pretrained medical undergraduates in the third year of training, and MBBS (Batchelor of Medicine and Batchelor of Surgery) qualified medical officers

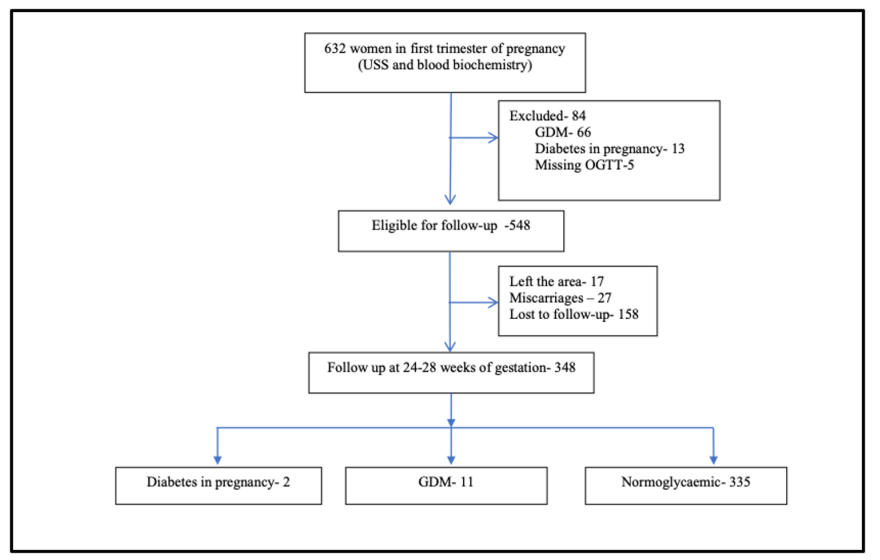

Figure 1 Study flow chart of the participants recruited and loss to follow-up. GDM, gestational diabetes mellitus; OGTT, oral glucose tolerance test; USS, ultrasound scan. 
performed clinical data collection and examination. Participants with a history of diabetes mellitus (DM) and currently on treatment for asthma, psychiatric diseases, autoimmune diseases, cardiovascular events, uncertain period of amenorrhea (POA), documented evidence of polycystic ovarian syndrome (PCOS), known liver diseases (except NAFLD), and history of using steatogenic drugs were excluded from this study.

Blood pressure was recorded using a high precision automated blood pressure measuring instrument (Omron Corporation) as the mean of two readings taken 5 min apart from both arms and was categorised as normal and high values for the first and second trimesters according to the National Institute for Health and Care Excellence (NICE) guidelines 2019. ${ }^{27}$

Weight (Wt) was measured using a digital weighing scale, and height was measured using a portable stadiometer. Waist circumference (WC) was measured by placing a non-stretchable fibre-glass measuring tape around the waist midway between the last rib and iliac crest with the subject in the standing position. Hip circumference was measured as the maximum circumference of the buttocks. This entered data was standardised to ensure the routine data were of high quality. ${ }^{28}$ As the weight gain and changes in WC during the first trimester of pregnancy are minimal, we use standard calculation methods and ranges for the Asian adult population to calculate body mass index (BMI), cut-off levels of obesity, and WC pregnancy period.

Blood samples were collected using standard guidelines by qualified nurses. Prerequisites for sample collection were informed to pregnant women prior to the blood collection date. Venipuncture was performed at ante-cubital fossa under aseptic conditions and universal precautions. All these samples were analysed on the same day by an automated analyser (Mindray BS-240 clinical chemistry analyser). Internal quality control was performed before every analytical run. In addition, external quality control was done using peer comparison every month during sample collection and period of analysis of collected blood samples.

An oral glucose tolerance test (OGTT) was performed on all participants at the recruitment and second trimester. Diagnoses of diabetes mellitus in pregnancy (DIP) and GDM were performed using WHO (2016) criteria. ${ }^{29}$ GDM was defined as fasting blood sugar (FBS) of 5.1-6.9 mmol/L and/or second-hour plasma glucose of $8.5-11.1 \mathrm{mmol} / \mathrm{L}$ by a $75 \mathrm{~g}$ glucose test anytime in pregnancy. Those with FBS $\geq 7 \mathrm{mmol} / \mathrm{L}$ and/or second-hour plasma glucose $\geq 11.1 \mathrm{mmol} / \mathrm{L}$ were labelled as DIP. ${ }^{29}$ In addition, levels of serum aminotransferases, gammaglutamyl transferases, and serum lipid levels were done as baseline screening tests to identify any liver-related pathological conditions and pre-existing dyslipidaemia.

Ultrasound scan (USS) of the abdomen was performed by competent and qualified investigators using Phillips affinity 70G machine with grey scale, colour Doppler, power Doppler, and spectral Doppler capabilities with curvilinear array transducer in the range of $2-5 \mathrm{MHz}$. During the procedure, four to five ultrasound images were taken, and the diagnosis of fatty liver was made by a board-certified consultant in radiology on the same monitor under the same lighting conditions with level III ultrasonography evidence to minimise observer bias. At the time of scanning, the radiologist was blinded with patients' clinical and laboratory data and unaware of previous reports.

Liver echogenicity was compared with the ipsilateral renal cortex and the spleen. In addition, the attenuation of waves, loss of demarcation of the diaphragm, and poor demarcation of the intrahepatic architecture were examined. Thus, fatty liver was graded as follows: Grade 0: normal liver echogenicity; Grade I: diffusely increased hepatic echogenicity but periportal and diaphragmatic echogenicity is still appreciable; Grade II: diffusely increased hepatic echogenicity obscuring periportal echogenicity but diaphragmatic echogenicity is still appreciable; and Grade III: diffusely increased hepatic echogenicity obscuring periportal and diaphragmatic echogenicity. ${ }^{14}$

Although comparison of fatty liver between pregnancy and the general population is not a major objective of the study, a small sample of age-matched randomly selected females was subjected to USS to overcome the possible bias of early pregnancy liver changes.

\section{Follow-up}

All pregnant women with normoglycaemia were followed up, and an OGTT was performed in between 24 and 28 weeks of POA.

\section{Data analysis}

According to the FLG, all continuous variables were summarised as the means with SD. The discrete data were presented as medians. To determine the association between fatty liver grade versus hyperglycaemia and miscarriages, the unadjusted relative risk (RR) was calculated, and to determine independent predictors of outcomes, binary logistic regression was performed, and adjusted ORs were calculated.

\section{RESULTS}

\section{Baseline assessment}

Altogether, 632 pregnant women with POA $\leq 12$ weeks were recruited. The mean age of the sample was 28.5 (SD 5.8 ) years, and most of them $(55.2 \%)$ were in the age category of 21-30 years (table 1 ). The majority $(31.5 \%)$ of the mothers were in their second pregnancy and had completed post-primary education.

Although the diagnosis of DM was reported by only 6 women, 18 (including the 6 with DM) reported a history of hyperglycaemic detected once or more than once. Prevalence of self-reported hypertension $(36,5.9 \%)$, dyslipidaemias $(8,1.3 \%)$, hypothyroidism $(17,2.7 \%)$ was low. Of the multigravida women, a history of GDM was 
Table 1 Baseline characteristics of the 634 first trimester pregnant women recruited for NAFLD assessment

\begin{tabular}{lll}
\hline & & $\mathbf{n}(\%)$ \\
\hline Age (years) & $<20$ & $42(8.8)$ \\
& $20-24$ & $124(19.60$ \\
& $25-29$ & $204(32.2)$ \\
& $30-34$ & $160(25.2)$ \\
& $34-39$ & $91(14.4)$ \\
& $>39$ & $13(2.1)$ \\
\hline Ethnicity & Sinhala & $573(90.4)$ \\
& Moor/Malay & $53(8.4)$ \\
& Other & $891.3)$ \\
\hline Gravidity & 1 & $179(28.5)$ \\
& 2 & $197(31.5)$ \\
& 3 & $163(26.1)$ \\
& 4 or more & $87(13.9)$ \\
\hline Education level & Primary only & $9(1.4)$ \\
& Below ordinary & $74(11.7)$ \\
& level & $360(56.8)$ \\
& Ordinary level & $191(30.1)$ \\
& Advanced level & \\
\hline diabetes mellitus & Yes & $6(1.0 \%)$ \\
& No & $594(99 \%)$ \\
\hline
\end{tabular}

n, number. reported by $17(2.7 \%)$, and the history of having at least one miscarriage was $19.1 \%(\mathrm{n}=121)$.

Of the pregnant women recruited, 324 (51.2\%) had either fatty liver grade I (FLG I) ( $\mathrm{n}=234,37 \%, 95 \%$ CI $33 \%$ to $41 \%$ ) or fatty liver grade II (FLG II) $(90,14 \%$, $95 \%$ CI $12 \%$ to $17 \%$ ). None of the participants had fatty liver grade III (FLG III). The prevalence of FLG I and in the non-pregnant comparison group $(n=56)$ was $38 \%$ $(n=21)$ and $14 \%(n=4)$, respectively. There was no statistically significant difference between the two groups $\left(\chi^{2}\right.$ 0.006, $\mathrm{p}=0.997$ ).

All tested liver parameters, biophysical parameters, and OGTT second-hour values gradually increased across the fatty liver grades. The difference between fatty liver grades I and II was higher than the difference between grades 0 and $I$ in all parameters except the FBS value, minimum diastolic blood pressure, portal vein diameter, and dome-to-pole length. FBS in the first trimester was reduced gradually across the FLGs (table 2 ).

Of the participants recruited, 84 were excluded from the follow-up study due to detection of DIP $(13,2.1 \%)$, $\operatorname{GDM}(66,10.4 \%)$, and missing OGTT values $(5,0.8 \%)$. Of the 548 eligible participants, 17 (3.1\%) pregnant women left the area, and from the rest, $27(5.1 \%)$ had late first-trimester or second-trimester miscarriages (figure 1).

Of the rest, 348 pregnant women were assessed at the end of the second trimester. Two cases of DM in pregnancy and 11 GDM cases were detected among

Table 2 Comparison of liver parameters, plasma glucose, and biophysical measurements by fatty liver grades among 632 first trimester pregnant women

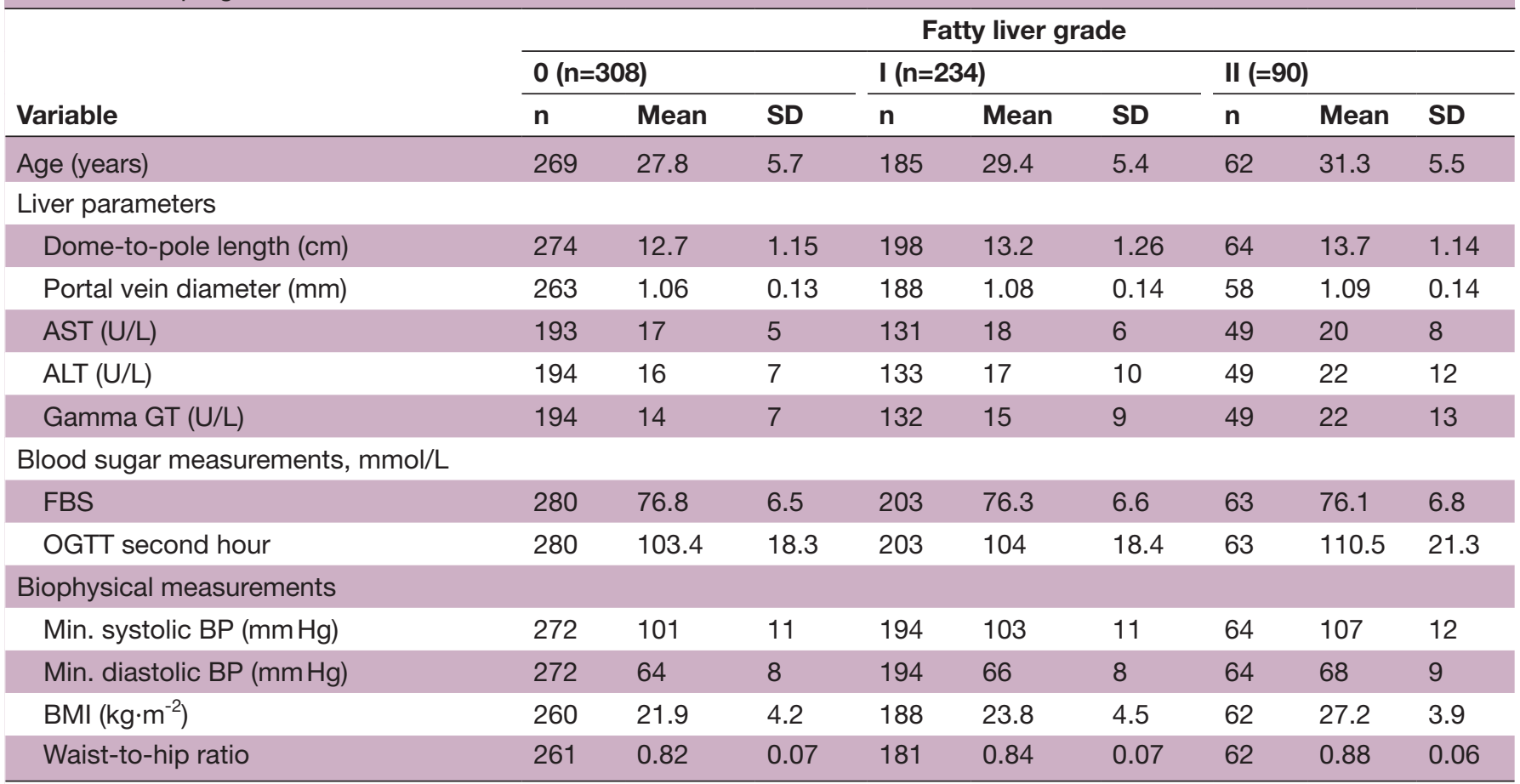

ALT, alanine aminotransferas; AST, aspartate aminotransferase; BMI, body mass index; FBS, fasting blood sugar; gamma GT, gammaglutamyl transferase; min. diastolic BP, minimum diastolic blood pressure; min. systolic BP, minimum systolic blood pressure; $n$, number; OGTT, oral glucose tolerance test. 


\begin{tabular}{|c|c|c|c|}
\hline Factor & $\begin{array}{l}\text { Adjusted } \\
\text { OR }\end{array}$ & $95 \% \mathrm{Cl}$ & $\begin{array}{l}\text { Significance } \\
\text { (P-value) }\end{array}$ \\
\hline Fatty liver grade II & 12.3 & 2.2 to 66.4 & 0.003 \\
\hline Fatty liver grade I & 3.3 & 0.6 to 18.7 & 0.166 \\
\hline Age & 0.9 & 0.3 to 2.2 & 0.829 \\
\hline $\begin{array}{l}\text { Parity (primi over } \\
\text { multigravida) }\end{array}$ & 2.5 & 0.6 to 10.2 & 0.191 \\
\hline BMI & 1.4 & 0.7 to 2.8 & 0.272 \\
\hline Waist-to-hip ratio & 0.8 & 0.3 to 1.6 & 0.579 \\
\hline $\begin{array}{l}\text { Family history of } \\
\text { diabetes }\end{array}$ & 0.6 & 0.1 to 3.5 & 0.642 \\
\hline Female education & 0.7 & 0.5 to 1.1 & 0.170 \\
\hline
\end{tabular}

BMI, body mass index; DM, diabetes mellitus; GDM, gestational diabetes mellitus.

previously normoglycaemic women. The incidence of DIP/GDM of FLG 0, I, and II in the second trimester was 11, 44, and 162 per 1000 pregnancies, respectively. The unadjusted RR of developing DIP/GDM in the second trimester compared with FLG 0 was 3.8 (95\% CI 0.79 to 19.4 ) and 12.5 (95\% CI 2.6 to 60.0) for FLG I and FLG II, respectively (table 3 ). The unconfounded effect of FL on developing GDM/DIP was assessed using a binary logistic regression model by adjusting for waist-to-hip ratio, age, parity, FLG, family history of diabetes, female education, and BMI as probable confounders. Only the FLG II has emerged as a significant predictor of DIP/GDM in second pregnancy with an OR of 12.3 (table 3).

Among 12 pregnant women with GDM (and had BMI assessment), 10 had higher BMI values (sensitivity $83.3 \%$ ), while 5 had FLG II (sensitivity $41.6 \%$ ). Out of the five patients with GDM with FLG II, four could be identified by BMI level (>22.9). However, 10 patients with GDM were identified after screening 151 participants with higher BMI (incidence 66.2 per 1000), while 6 mothers with GDM were identified after screening for only 43 participants with FLG II (incidence 139.5 per 1000).

The cumulative incidences of miscarriages among FLG 0 , I, and II were 35, 76, and 159 per 1000 pregnancies. Compared with those with FLG 0, pregnant women with FLG I had more than two times the risk (RR 2.1, 95\% CI 1.01 to 4.64 ) of miscarriage. Similarly, FLG II had more than four times the risk of miscarriage $(4.5,95 \%$ CI 2.1 to 9.9) compared with FLG 0. The adjusted OR for FLG II compared with both FLG 0 and FLG I was calculated after adjusting for age, parity, blood pressure, blood sugar, haemoglobin, and BMI, and FLG II remained the only independent predictor of miscarriages with a higher level of statistical significance even after adjusting for the above confounding factors (adjusted OR 4.2, 95\% CI (1.9 to 9.1 ).

\section{DISCUSSION AND INTERPRETATION}

NAFLD during pregnancy partially reflects the physiological changes with fluctuation of oestrogen, lipid levels, and rapid weight gain during pregnancy. ${ }^{30}$ Nevertheless, the link between NAFLD and GDM has been studied in both directions. NAFLD in the first trimester has shown to be a risk factor for dysglycemia in mid-pregnancy, ${ }^{9}$ and having gestational diabetes was also identified as a risk factor for postpartum development of NAFLD. ${ }^{31}$ This study indicates that the prevalence of fatty liver among pregnant women in this rural South Asian community is higher than the reported values from high-income countries (HICs) and the previously reported in the South Asian context. Strengthening the evidence generated elsewhere, ${ }^{32} 33$ this prospective study indicates that NAFLD, specifically FLG II is a major predictor of the development of GDM in pregnancy. Although this association is reported in HICs, ours is the first study to reflect this in the rural Asian population using a communitybased prospective cohort study. Even though a previous study reports that NAFLD may not be a significant risk factor for diabetes after adjusting for BMI and age, ${ }^{9}$ our study shows that the FLG II is the only predictor of GDM/DIP, even after adjusting for those confounding variables. The diagnostic thresholds and criteria for GDM are different across the globe and are evolving. The strength of the association in this study is difficult to compare directly with many previous studies due to the differences in GDM diagnostic criteria. De Souza et al used similar criteria for GDM and revealed an adjusted OR ranging from 6.8 to $7.8 .^{32}$ Mousa $e t$ al also reported a significantly higher incidence of GDM among pregnant mothers with fatty liver. ${ }^{9}$ Our estimates are much larger yet have wide confidence intervals (similar to the study by De Souza et al).

Although high BMI is a well-recognised risk factor for NAFLD worldwide, the prevalence of NAFLD among low BMI people (lean NAFLD) was high among Asian people than West. ${ }^{34}$ According to our results, the sensitivity of detecting NAFLD is high with a higher BMI $(83.3 \%)$. However, predictive value of a positive test is very low, making it a poor predictor which was reflected in the logistic regression model. Nevertheless, the number of women with the outcome GDM was inadequate to discuss the higher prevalence of lean NAFLD in this population.

The observed NAFLD prevalence of $51.3 \%$ in early pregnancy seems higher than the previously reported values for Sri Lanka (18.2\%), ${ }^{14}$ Canada $(17.6 \%),{ }^{10}$ Korea $(18.4 \%),{ }^{13} 35$ and the USA $(14.3 \%-16.7 \%) .{ }^{12}$ However, the prevalence in this study is not significantly different from the comparison group of non-pregnant reproductive-age women from the same geographical location. ${ }^{36}$ Therefore, irrespective of pregnancy, this high prevalence of fatty liver in this rural young women (mean age 28.8 years) needs to be taken seriously as a major predictor of future non-communicable diseases (NCDs).

NAFLD is considered a hepatic manifestation of metabolic syndrome. ${ }^{37}$ Other than GDM, one of the most important findings of this study was the unprecedented observation of FL as a major risk factor for miscarriages with an adjusted OR of 4.2. Liu et alshowed that abortions 
in women with NAFLD were higher than those without NAFLD $(72.4 \%$ vs $69.3 \%$, age-adjusted $\mathrm{p}=0.001) .{ }^{16}$ The pathophysiology behind this association is still not very clear. However, we can assume that oxidative stress, endothelial damage, and inflammation may predispose to the development of early pregnancy loss. ${ }^{38}$ However, fatty liver is a common finding among PCOS women of reproductive age.$^{39}$ Insulin resistance, growing epidemics of obesity, and androgen excess may contribute to the development of NAFLD among PCOS. ${ }^{39}$ Therefore, the association between fatty liver and miscarriages could be due to PCOS. ${ }^{40}$ Further studies should be planned to determine the association between PCOS, fatty liver, and miscarriages. These observations with a high effect size are of significant public health importance in predicting miscarriages and probably formulating new guidelines for pregnancy care programmes.

Current evidence on diabetes clearly shows that South Asians are at an increased risk of all metabolic derangements, including NAFLD, DM, and GDM. ${ }^{41}$ Combining this knowledge with public health service provisions could be a practical and comprehensive approach to pregnancy care programmes. USS is use for many years as a part of routine pregnancy care, as a 'dating scan.' Thus, a simple added step in dating scans to screen for fatty liver during pregnancy may show the risk of developing hyperglycaemia and mid-pregnancy miscarriage. Our multivariable analysis shows that NAFLD is a better predictor of these conditions than the traditional 'risk factors' considered in pregnancy. Early identification, monitoring, and intervention for high-risk pregnant women using USS will be a non-invasive screening procedure that could be easily integrated into the system. In addition, the diagnosis of NAFLD in pregnant women would be an early life opportunity to predict and prevent future NCDs among these women.

\section{Limitations}

We used USS to diagnose NAFLD in this study instead of the gold standard liver biopsy. USS is a non-invasive, acceptable, and feasible alternative method, especially in pregnant women. The sensitivity of the diagnosis of fatty liver in USS ranges from $60 \%$ to $94 \%$, and the specificity is from $84 \%$ to $95 \%$. The sensitivity of detecting fatty liver is increased when the degree of fatty liver increases. In morbid obesity, sensitivity and specificity are reduced from $49 \%$ to $75 \%$ due to technical errors. However, evidence shows that the reliability of USS for diagnosing fatty liver is higher than that of histology in people with moderate and severe fatty liver. In addition, the grading of fatty liver is subjective and operator-dependent. ${ }^{14}$ The objective of the FL scan in our study is risk prediction rather than a precise diagnosis of steatosis, and for that purpose, routine USS will be adequate.

We used the latest WHO criteria ${ }^{42}$ for diagnosing GDM with an OGTT in the first trimester, which led to the exclusion of 66 pregnant women from the baseline assessment who should not be excluded if the previous classification was used. Since the assessment of glycaemic level is usually done in the second trimester, these pregnant women may not have been excluded in the usual analysis. This exclusion has led to wide confidence intervals showing the need for a larger sample size. However, the estimated risk is the absolute risk of developing hyperglycaemic in the second trimester among those without having any form of hyperglycaemic in the first trimester.

\section{CONCLUSION}

FLG II, diagnosed by a simple USS, is a major predictor of GDM and early pregnancy miscarriage. The prevalence of NAFLD is higher than that reported elsewhere and in the same context in the past. Therefore, pre-conceptional and/or early pregnancy diagnosis of NAFLD probably incorporated into the routine USS in pregnancy should be considered to identify this important risk factor early. In addition, we recommend incorporating the USS scan to detect fatty liver with the routine dating scan of pregnancy so that additional preventive healthcare could be provided for the mothers having grade 2 fatty liver.

\section{Author affiliations}

${ }^{1}$ Department of Anatomy, Faculty of Medicine and Allied Sciences, Rajarata University of Sri Lanka, Saliyapura, North Central, Sri Lanka

${ }^{2}$ Department of Community Medicine, Faculty of Medicine and Allied Sciences, Rajarata University of Sri Lanka, Saliyapura, North Central, Sri Lanka

${ }^{3}$ Maternal and Child Health Research Unit, Faculty of Medicine and Allied Sciences, Rajarata University of Sri Lanka, Saliyapura, North Central, Sri Lanka

${ }^{4}$ Department of Radiology, Teaching Hospital Anuradhapura, Anuradhapura, North

Central, Sri Lanka

${ }^{5}$ Department of Obstetrics and Gynaecology, Teaching Hospital Anuradhapura, Anuradhapura, North Central, Sri Lanka

Acknowledgements We acknowledge all participant mothers, data collectors, field staff in Anuradhapura regional director of health services division and the Medical Officer of Maternal and Child Health Anuradhapura. This study was conducted as a part of a large cohort study (RaPCo), and we acknowledge Professor Thilini Agampodi (principal investigator) (PI), Dr Nuwan Wickramasinghe (Co-PI), and all other investigators from the original study.

Contributors Conceptualisation, designing the study, and data analysis were done by ISK, JNW, and SBA. ISK, KGD, JNW, and FNDS did the data curation, while SBA made funding acquisitions. Experimental procedures were done by ISK, KGD, and FNDS. All authors were involved in writing the article, while SBA and KGD were involved in supervision. ISK is responsible for the overall content as the guarantor.

Funding This study was funded through the Accelerating Higher Education Expansion and Development (AHEAD) grant (grant number: DOR STEM HEMS (6026-LK/8743-LK)), a World Bank-funded project through the University grant commission Sri Lanka.

Competing interests None declared.

Patient consent for publication Not applicable.

Ethics approval The ethical clearance was obtained from the Ethics Review Committee of the Faculty of Medicine and Allied Sciences, the Rajarata University of Sri Lanka under ERC/2019/22 and approved on 15/08/2019. Written informed consent was taken from all pregnant mothers to participate in this study and to use routinely collected data for this research purpose. The participants were informed that this research is conducted in parallel with the routine maternal and child health service, and any 'abnormality' will be reported to the health provider with the participant's consent. In addition, consent had been sought to use a serum sample to screen diabetes mellitus, liver biochemistry and serum cholesterol level, and any other future studies that may require baseline assessment of serum.

Provenance and peer review Not commissioned; externally peer reviewed. 
Data availability statement Anonymous data used in this study will be available from the first author for the researcher on a reasonable request.

Supplemental material This content has been supplied by the author(s). It has not been vetted by BMJ Publishing Group Limited (BMJ) and may not have been peer-reviewed. Any opinions or recommendations discussed are solely those of the author(s) and are not endorsed by BMJ. BMJ disclaims all liability and responsibility arising from any reliance placed on the content. Where the content includes any translated material, BMJ does not warrant the accuracy and reliability of the translations (including but not limited to local regulations, clinical guidelines, terminology, drug names and drug dosages), and is not responsible for any error and/or omissions arising from translation and adaptation or otherwise.

Open access This is an open access article distributed in accordance with the Creative Commons Attribution 4.0 Unported (CC BY 4.0) license, which permits others to copy, redistribute, remix, transform and build upon this work for any purpose, provided the original work is properly cited, a link to the licence is given, and indication of whether changes were made. See: https://creativecommons.org/ licenses/by/4.0/.

ORCID iD

Iresha Sandamali Koralegedara http://orcid.org/0000-0001-5479-8554

\section{REFERENCES}

1 Younossi Z, Anstee QM, Marietti M, et al. Global burden of NAFLD and NASH: trends, predictions, risk factors and prevention. Nat Rev Gastroenterol Hepatol 2018;15:11-20.

2 Basaranoglu M, Neuschwander-Tetri BA. Nonalcoholic fatty liver disease: clinical features and pathogenesis. Gastroenterol Hepatol 2006;2:282-91.

3 Prins GH, Luangmonkong T, Oosterhuis D, et al. A pathophysiological model of non-alcoholic fatty liver disease using precision-cut liver slices. Nutrients 2019;11:507.

4 Fan J-G, Kim S-U, Wong VW-S. New trends on obesity and NAFLD in Asia. J Hepatol 2017;67:862-73.

5 Vernon G, Baranova A, Younossi ZM. Systematic review: the epidemiology and natural history of non-alcoholic fatty liver disease and non-alcoholic steatohepatitis in adults. Aliment Pharmacol Ther 2011;34:274-85.

6 VanWagner LB, Armstrong MJ. Lean NAFLD: a not so benign condition? Hepatol Commun 2018;2:5-8.

7 Page LM, Girling JC. A novel cause for abnormal liver function tests in pregnancy and the puerperium: non-alcoholic fatty liver disease. BJOG 2011;118:1532-5.

8 Mclntyre HD, Catalano P, Zhang C, et al. Gestational diabetes mellitus. Nat Rev Dis Primers 2019;5:1-19.

9 Mousa N, Abdel-Razik A, Shams M, et al. Impact of non-alcoholic fatty liver disease on pregnancy. Br J Biomed Sci 2018;75:197-9.

10 De Souza LR, Berger H, Retnakaran R, et al. Non-Alcoholic fatty live disease in early pregnancy predicts dysglycemia in mid-pregnancy: prospective study. Am J Gastroenterol 2016;111:665-70.

11 Sattari M, Bril F, Egerman R, et al. Relationship between nonalcoholic fatty liver disease during pregnancy and abnormal glucose metabolism during and after pregnancy. J Investig Med 2020;68:743-7.

12 Sarkar M, Djerboua M, Flemming JA. Nafld cirrhosis is rising among childbearing women and is the most common cause of cirrhosis in pregnancy. Clin Gastroenterol Hepatol 2022;20:e315-8.

13 Lee SM, Kwak SH, Koo JN, et al. Non-Alcoholic fatty liver disease in the first trimester and subsequent development of gestational diabetes mellitus. Diabetologia 2019;62:238-48.

14 Herath HMM, Kodikara I, Weerarathna TP, et al. Prevalence and associations of non-alcoholic fatty liver disease (NAFLD) in Sri Lankan patients with type 2 diabetes: A single center study. Diabetes Metab Syndr 2019;13:246-50.

15 Sarkar M, Grab J, Dodge JL, et al. Non-Alcoholic fatty liver disease in pregnancy is associated with adverse maternal and perinatal outcomes. J Hepatol 2020;73:516-22.

16 Liu Y, Lu J, Xu M, et al. Association between history of abortion and nonalcoholic fatty liver disease in middle-aged and elderly Chinese women. Ann Epidemiol 2013;23:119-23.

17 Ajmera VH, Gunderson EP, VanWagner LB, et al. Gestational diabetes mellitus is strongly associated with non-alcoholic fatty liver disease. Am J Gastroenterol 2016;111:658-64.
18 Foghsgaard S, Andreasen C, Vedtofte L, et al. Nonalcoholic fatty liver disease is prevalent in women with prior gestational diabetes mellitus and independently associated with insulin resistance and waist circumference. Diabetes Care 2017;40:109-16.

19 The Role of Non-Alcoholic Fatty Liver Disease in Gestational Diabetes - Full Text View - ClinicalTrials.gov. Available: https:// clinicaltrials.gov/ct2/show/NCT01855386 [Accessed 30 Jan 2019].

20 NAFLD in pregnancy increases risks for mother and baby - EASLThe Home of Hepatology. Available: https://easl.eu/press-release/ nafld-pregnancy-risks/ [Accessed 8 Feb 2021]

21 Rathi U, Bapat M, Rathi P, et al. Effect of liver disease on maternal and fetal outcome--a prospective study. Indian J Gastroenterol 2007;26:59-63.

22 Behboudi-Gandevani S, Amiri M, Bidhendi Yarandi R, et al. The impact of diagnostic criteria for gestational diabetes on its prevalence: a systematic review and meta-analysis. Diabetol Metab Syndr 2019;11:11:11.

23 Mi Lee S, Shin Park J, Norwitz ER. Nonalcoholic fatty liver disease in the first trimester as a risk factor for large-for-gestational-age birthweight: a prospective study 2018.

24 Agampodi TC, Wickramasinghe ND, Prasanna RIR, et al. The Rajarata pregnancy cohort (RaPCo): study protocol. BMC Pregnancy Childbirth 2020;20:374.

$25 \mathrm{MOMCH}$ Unit. Regional director of health services, Anuradhapura SL. annual statistics, 2019.

26 Paul HA, Collins KH, Nicolucci AC, et al. Maternal prebiotic supplementation reduces fatty liver development in offspring through altered microbial and metabolomic profiles in rats. Faseb $J$ 2019;33:5153-67.

27 NICE. Overview | Hypertension in pregnancy: diagnosis and management | Guidance |. Available: https://www.nice.org.uk guidance/cg107 [Accessed 26 Apr 2019]

28 Management of obesity. Available: https://www.researchgate.net/ publication/301302217_Management_of_obesity [Accessed 9 Mar 2019]

29 WHO recommendations on antenatal care for a positive pregnancy experience.

30 Lao TT. Implications of abnormal liver function in pregnancy and non-alcoholic fatty liver disease. Best Pract Res Clin Obstet Gynaecol 2020;68:2-11.

31 Le MH, Devaki P, Ha NB, et al. Prevalence of non-alcoholic fatty liver disease and risk factors for advanced fibrosis and mortality in the United States. PLoS One 2017;12:e0173499.

32 De Souza LR, Berger H, Retnakaran R, et al. Hepatic fat and abdominal adiposity in early pregnancy together predict impaired glucose homeostasis in mid-pregnancy. Nutr Diabetes 2016;6:e229.

33 Herath RP, Siriwardana SR, Ekanayake CD, et al. Non-Alcoholic fatty liver disease and pregnancy complications among Sri Lankan women: a cross sectional analytical study. PLoS One 2019:14:e0215326.

34 Lu F-B, Zheng KI, Rios RS, et al. Global epidemiology of lean nonalcoholic fatty liver disease: a systematic review and meta-analysis. J Gastroenterol Hepatol 2020;35:2041-50.

35 Hagström H, Höijer J, Ludvigsson JF, et al. Adverse outcomes of pregnancy in women with non-alcoholic fatty liver disease. Liver Int 2016;36:268-74.

36 Chai TYL, Rajaratnam RM, Deng D, et al. The prevalence of gestational diabetes mellitus in women diagnosed with non-alcoholic fatty liver disease during pregnancy: a systematic review and metaanalysis. J Diabetes Complications 2021;35:107991.

37 Gastaldelli A. Fatty liver disease: the hepatic manifestation of metabolic syndrome. Hypertens Res 2010;33:546-7.

38 Buzzetti E, Pinzani M, Tsochatzis EA. The multiple-hit pathogenesis of non-alcoholic fatty liver disease (NAFLD). Metabolism 2016;65:1038-48.

39 Vassilatou E. Nonalcoholic fatty liver disease and polycystic ovary syndrome. World J Gastroenterol 2014;20:8351.

40 Al-Jaroudi D. Non-Alcoholic fatty liver disease in infertile women with polycystic ovarian syndrome: a prospective series. Int J Womens Health Wellness 2017;3:047.

41 Misra A, Khurana L. The metabolic syndrome in South Asians: epidemiology, determinants, and prevention. Metab Syndr Relat Disord 2009;7:497-514.

42 International Association of Diabetes and Pregnancy Study Groups Consensus Panel, Metzger BE, Gabbe SG, et al. International association of diabetes and pregnancy study groups recommendations on the diagnosis and classification of hyperglycemia in pregnancy. Diabetes Care 2010;33:e98-82. 